\title{
Terapia Ocupacional e o cuidado integral a saúde de crianças e adolescentes: a construção do Projeto ACCALANTO*
}

\section{Occupational Therapy and the integral health care of children and adolescents: the construction of the Project ACCALANTO}

\author{
Sandra Maria Galheigo', Andréa do Amparo Carotta de Angeli²
}

GALHEIGO, S. M.; ANGELI, A. A. C. de. Terapia Ocupacional e o cuidado integral a saúde de crianças e adolescentes: a construção do Projeto ACCALANTO. Rev. Ter. Ocup. Univ. São Paulo, v. 19, n. 3, p. 137-143, set./dez. 2008.

RESUMO: Este artigo se propõe a apresentar o Projeto ACCALANTO, que desenvolve atividades de ensino, pesquisa, extensão no âmbito da terapia ocupacional e da saúde da criança e do adolescente, sob a perspectiva da integralidade e humanização do cuidado e da proteção integral da infância e da juventude. Esse projeto se propõe a contribuir para a construção de práticas e saberes de Terapia Ocupacional apoiados nos princípios de uma clínica ampliada que reconhece e acolhe as necessidades singulares da criança, do adolescente e suas famílias/cuidadores, ocasionadas pelo processo de adoecimento e hospitalização e pelas condições de vulnerabilidade social a que estão submetidos. O artigo apresenta as justificativas e norteadores teórico-metodológicos do Projeto ACCALANTO assim como seus principais eixos e estratégias de cuidado numa enfermaria pediátrica, a saber: (i) o Ninar - escuta e acolhimento às necessidades das crianças de 0 a 2 anos e de seus pais, buscando desenvolver ações de promoção ao cuidado numa dimensão ampliada; (ii) o Aquarela - cuidado de crianças e adolescentes cujas condições de saúde são marcadas pela intensidade, cronicidade ou gravidade dos acometimentos que sofrem, demandando intervenções particularizadas com grau elevado de planejamento e controle; (iii) o Amarelinha - propiciar uma convivência e cotidianidade diferenciada no espaço hospitalar através de atividades que podem ser oferecidas ao conjunto de usuários na brinquedoteca e nas áreas de convivência da enfermaria. (iv) o AdoleSer - ações voltadas à população adolescente em geral e a jovens mães, ofertando um espaço de re-invenção de si e de projetos de vida.

DESCRITORES: Assistência integral à saúde. Terapia ocupacional. Serviço hospitalar de terapia ocupacional. Cuidado da criança. Processo saúde-doença. Atividades cotidianas.

* Artigo produto de projeto de extensão Projeto ACCALANTO - Atividade, cotidiano e cuidado: terapia ocupacional e saúde integral da criança e do adolescente, que recebeu apoio do Fundo de Cultura e Extensão da Universidade de São Paulo em 2008.

1 Professora doutora, docente do Departamento de Fisioterapia, Fonoaudiologia e Terapia Ocupacional da Faculdade de Medicina da USP.

2 Terapeuta ocupacional, técnica do Departamento de Fisioterapia, Fonoaudiologia e Terapia Ocupacional da Faculdade de Medicina da USP.

Endereço para correspondência: Centro de Docência e Pesquisa em Terapia Ocupacional da FMUSP, Rua Cipotânea, 51 - Cidade Universitária, CEP: 05508-900, São Paulo-SP. 


\section{INTRODUÇÃO}

$\mathrm{O}$ Projeto ACCALANTO ${ }^{(1)}$ - Atividade, cotidiano e cuidado: terapia ocupacional e saúde integral da criança e do adolescente - é um projeto que desenvolve atividades de ensino, pesquisa, extensão no âmbito da terapia ocupacional e da saúde da criança e do adolescente, sob a perspectiva da integralidade e humanização do cuidado e da proteção integral da infância e da juventude, em consonância com o Estatuto da Criança e do Adolescente e as proposições do Ministério da Saúde, a saber: a Agenda de Compromissos para a Saúde Integral da Criança e Redução da Mortalidade Infantil; Saúde integral de adolescentes e jovens: orientações para a organização de serviços de saúde e a Política Nacional de Humanização (PNH) (Brasil. 2004a, b, 2005). O projeto, assim, se propõe a contribuir para a construção de práticas e saberes de Terapia Ocupacional apoiados nos princípios de uma clínica ampliada que reconhece e acolhe as necessidades singulares da criança, do adolescente e suas famílias/cuidadores, ocasionadas pelo processo de adoecimento e hospitalização e pelas condições de vulnerabilidade social a que estão submetidos (GALHEIGO; ANGELI 2007).

Compreende que os processos saúde - doença - cuidado se manifestam na interação de diferentes elementos - corpo, subjetividade, adoecimento, atividade, cotidiano, práticas de saúde e contexto sócio-cultural - e, neste sentido, busca contribuir para a constituição de estratégias de cuidado e de enfrentamento das dificuldades vivenciadas por crianças, adolescentes e familiares/cuidadores, em suas ações e cotidianos. Entretanto, trata-se de um cuidado ampliado que busca, em paralelo, mapear as redes de suporte e proteção dos envolvidos, contribuindo para a construção e fortalecimento de uma atenção interdisciplinar, intersetorial e em rede.

Iniciou suas atividades em agosto de 2007 numa enfermaria pediátrica de um hospital geral, tendo como proposta a ampliação de suas atividades a outros equipamentos da rede de atenção à saúde, sejam ambulatórios, unidades básicas de saúde, ou demais equipamentos sociais, buscando assim percorrer-construir linhas de cuidado para a atenção a crianças e adolescentes.

No que se refere às atividades de ensino, o Projeto ACCALANTO está vinculado a cinco disciplinas da grade curricular do Curso de Terapia Ocupacional da
FMUSP, das quais duas são vinculadas à formação prática (Prática Supervisionada/ Estágio em Terapia Ocupacional e Contextos Hospitalares), uma ao aprofundamento teórico (Terapia Ocupacional em Programas Hospitalares) e outras duas ao trabalho iniciação do aluno à pesquisa.

A dimensão do ensino e da formação de terapeutas ocupacionais para o trabalho no hospital traz em seu bojo um conjunto de desafios. Na graduação, um dos mais importantes é o de formar profissionais que estejam capacitados para identificar, de forma compreensiva, as necessidades e demandas dos usuários e de sua rede social. Ademais, espera-se que, de posse dessas informações e do conjunto de tecnologias de cuidado adquiridas durante sua formação, estudantes consigam elaborar e implementar estratégias de ação sensíveis, pertinentes e contextualizadas. Buscando-se, desta forma, evitar a formação precoce para a especialidade, assim como, a construção de um perfil profissional que domine apenas a realização de um conjunto de procedimentos ou técnicas utilizados em situações específicas, mas que não dialogue com o conjunto das necessidades das pessoas atendidas.

Nas práticas destinadas às pessoas sob cuidados clínicos e cirúrgicos, o ensino tem, ainda, como desafio desconstruir a centralidade do 'hospital das especialidades' no imaginário do estudante e recolocar a atenção da terapia ocupacional no hospital contemporâneo, a partir do lugar da produção do cuidado em saúde, com destaque para a dimensão da humanização e da integralidade do cuidado, conforme proposta pelo Sistema Único de Saúde (GALHEIGO, 2008). Deve possibilitar ao estudante vivenciar a prática da integralidade por meio da conjugação dos esforços dos diferentes profissionais e da construção de um diálogo permanente. Simultaneamente, a integralidade do cuidado vai sendo construída por meio da articulação das ações no hospital com as da rede de serviços, já que só pode ser pensada e realizada de forma efetiva se articulada em rede (CECÍLIO; MERHY, 2003; FEUERWERKER; CECÍLIO, 2007). Tem sido a partir desses pressupostos de formação profissional para a produção do cuidado em saúde que o projeto descrito a seguir vem sendo construído e desenvolvido.

\section{Justificativa e bases teórico-metodológicas do Projeto ACCALANTO}

A situação de internação hospitalar é pensada, aqui,

\footnotetext{
(1) O projeto obteve ao longo do ano de 2008, o suporte do Fundo de Cultura e Extensão (FCEx) da Universidade de São Paulo, contando com o trabalho de uma bolsista. Cabe ressaltar, que entre 2007-8, pode contar com o trabalho de duas bolsistas Coseas/USP que auxiliaram bastante na montagem da assistência. E, atualmente, conta com uma bolsista do Programa Aprender com Extensão.
} 
a partir das rupturas que promove na vida cotidiana e na apropriação do viver, dos corpos dos sujeitos que são atendidos. Quando sujeito de uma internação, por conta de um agravamento de seu processo de adoecimento e pela necessidade de cuidados de maior complexidade, vive-se um progressivo afastamento das condições de vida materiais e afetivas que constituíam um território existencial. As roupas são substituídas por aventais e pijamas, os rostos familiares são substituídos por desconhecidos que rapidamente se tornam "íntimos", a rotina é organizada em torno dos procedimentos médicos e de enfermagem, prioritariamente, o dia e a noite, passam a ser regrados pelos horários de medicamentos e/ou procedimentos que visam assegurar o prosseguimento da vida. A vida orgânica passa a ser objeto de cuidado dos ditos, profissionais de saúde.

Concordamos com Denise Sant'Anna (2001) ao dizer que

Ao transtorno de viver dependente dos cuidados alheios, pode-se somar aquele provocado pela quebra de conexão entre práticas que, na vida do indivíduo fora do hospital, possuem alguma continuidade: o corpo do hospitalizado transformase no principal lugar de manipulações descontínuas e fragmentadas; ele é freqüentemente tratado por partes e abstraído através de exames e fichas de cadastramento; a subjetividade do paciente é reduzida a identificação de elementos corporais (...) passíveis de mensuração e avaliação científica. $\mathrm{O}$ indivíduo se torna divisível na medida em que a intimidade de seu organismo é exposta dia e noite. (p. 32).

Neste sentido, pode-se pensar que para as crianças e adolescentes, o impacto da internação pode vir a gerar outras dificuldades, tendo em vista, sua condição particular de pessoas em processo de crescimento, sua dependência do cuidado do outro, o pouco repertório para lidar com a infinidade de situações e exposições a que estão submetidos na internação. Somam-se a isto, todas as demandas oriundas do próprio adoecimento.

Condição, ainda, particular da internação de crianças e adolescentes, é a presença, muitas vezes, obrigatória de familiares e/ou cuidadores que se revezam ou não na administração dos cuidados, na espera dos procedimentos e notícias, na espera do que podem ou não fazer durante o período que ficam no hospital. Expostos, de outro modo, são constantemente avaliados em sua capacidade de acalantar, de estar presentes, de cooperar com a equipe de saúde, de agüentar e entender todos os procedimentos, dentre outros. Em paralelo, essa demanda dificulta a realização de atividades de manutenção das condições de vida (seja da rotina doméstica ou de trabalho), do cuidado de seus outros dependentes, requerendo uma re-acomodação dos projetos pessoais e familiares. Nos casos em que o processo de adoecimento e hospitalização torna-se longo ou freqüente, o cansaço e a tensão decorrentes das responsabilidades tornam-se ainda maiores.

Entende-se que a ação da terapia ocupacional, nestas condições, se constitui em poder entender e intervir nas manifestações e descontinuidades da cotidianidade ocasionadas por situações diversas de adoecimento, pelo próprio impacto dos processos saúde-doença nos contextos de vida dos sujeitos e pela hospitalização. Cabe ressaltar que a noção de cotidiano, aqui, alude ao conjunto de afazeres que constituem o universo ocupacional das pessoas, isto é, das atividades costumeiras do dia-a-dia a aquelas que atribuem significados essenciais do viver do sujeito e de seu grupo social. Indo além, o cotidiano remete às redes de relações sociais e também, ao próprio modo de ser, estar e fazer de um coletivo.

Por meio do uso de diferentes ações oriundas do cotidiano da criança e do adolescente o terapeuta vem a oferecer um espaço para a experimentação de si e do outro. Produzindo um ambiente onde os sujeitos do cuidado possam atuar de forma a se apropriarem de suas produções de saúde, fomentando diálogos entre a compreensão e a vivência dos processos de saúde-doença e as leituras dos profissionais de saúde.

A produção de saúde, aqui, é compreendida pelo permanente movimento de invenção de si e do mundo, de modo que as perdas, as rupturas e os processos de adoecimento possam ser vividos como parte da processualidade própria do viver. Neste sentido, o terapeuta pode problematizar, vivenciar, cuidar com a pessoa sob sua atenção da expressão de seus desejos, angústias, ansiedades mapeando seus limites e potencialidades. Assim como, vislumbrar redes de apoio a situações de maior vulnerabilidade, ampliando deste modo sua autonomia.

Pode-se pensar, ainda, usando a construção de Mehry (1997a, b) que temos uma terapia ocupacional na produção do cuidado à saúde da criança e do adolescente que se inscreve, prioritariamente, no lugar das tecnologias leves, que se desenvolvem no espaço intersubjetivo do profissional de saúde e do sujeito sob cuidado. Tecnologias, estas, que são relacionais que envolvem a escuta, o vínculo, a singularização, dentre outras. E que se diferenciam significativamente dentro da rotina hospitalar dos procedimentos operados pelos demais profissionais. Entretanto, o terapeuta ocupacional também pode vir a fazer uso, do que os autores chamam de tecnologias leve-duras, que abarcariam os saberes clínicos, epidemiológicos, terapêuticos; e, em menor escala, das tecnologias duras, que abrangem a utilização de equipamentos de maior complexidade na assistência. Para Castro (2005), 
Este amplo exercício de leitura e compreensão de passagens clínicas revela as estratégias arrojadas de manejo clínico que as práticas da terapia ocupacional na contemporaneidade muitas vezes exigem e a qualidade da presença humana nestas funções (p.7).

O projeto ACCALANTO, assim, busca atuar em duas dimensões simultaneamente, num movimento 'para dentro' e 'para fora'. O primeiro foca no micro contexto das redes relacionais e de ação dentro hospital, promovendo a atenção às necessidades das crianças, adolescentes e seus cuidadores e o desenvolvimento da ambiência dos espaços, de modo reflexivo e em consonância com a Política Nacional de Humanização. O segundo busca estender sua ação ao macro contexto das redes relacionais fora do hospital, ou seja, nos territórios de vida dos sujeitos articulando-as em torno da sustentação do cuidado.

\section{Eixos e estratégias de Cuidado do Projeto ACCALANTO}

No semestre anterior à concepção do Projeto ACCALANTO, as atividades de prática e estágio supervisionados foram iniciadas na enfermaria de pediatria com a proposta central de conhecer sua estrutura física e organizacional e sua dinâmica de funcionamento; seus recursos humanos e materiais; os usuários do serviço e sua rede social, sua proveniência e condições sócioeconômicas;e, os motivos, período e freqüência de internação e as doenças mais comuns. As informações foram coletadas pelo acesso ao sistema de dados do hospital, aos prontuários e por entrevista com alguns membros da equipe. Ao mesmo tempo, as idas semanais à enfermaria possibilitaram coletar dados diretamente com os usuários, sua família e cuidadores e equipe. Ao mesmo tempo em que foi possível iniciar intervenções pontuais que contribuíram com novos elementos para a análise.

Em síntese, o estudo revelou que os recursos físicos da enfermaria eram de 10 quartos individuais com banheiro, 6 quartos coletivos com banheiro com 4 leitos em cada, 1 quarto coletivo de até 8 leitos, totalizando uma capacidade instalada de 40 leitos. Além disso, contava com dois postos de enfermagem, uma brinquedoteca que funciona das 8 às 16 horas, duas áreas comuns de lazer, com sofá, televisão e computador, sala de reunião e atendimento reservado, copa para funcionários além de outras dependências para administração, realização de procedimentos, higienização de materiais, dentre outros. Em relação à ambiência, a enfermaria mostrava-se bem cuidada, não se distanciando do habitual nas enfermarias dos hospitais públicos: luz florescente, paredes de cor clara, havendo desenhos infantis pintados em cores pastéis nas áreas comuns. A equipe permanente da enfermaria é composta pelos médicos, enfermeiros, técnicos de enfermagem, e uma pedagoga (encarregada da brinquedoteca). Conta regularmente com os serviços de fisioterapia, nutrição e farmácia do hospital e atendimento de psicologia provido por um laboratório de psicologia da universidade. $\mathrm{O}$ acesso a serviços de terapia ocupacional e fonoaudiologia acontece a partir de pedido de interconsulta. Cabe destacar que, as entrevistas com membros da equipe e a rotina hospitalar, evidenciaram uma compreensão limitada do papel do terapeuta ocupacional. Ao longo dos meses de coleta de dados, as interconsultas recebidas pela terapia ocupacional se limitavam ao pedido de confecção de órteses.

A enfermaria atende crianças e adolescentes de 0 a 14 anos, com a maior concentração de internados em seus primeiros cinco anos, sendo de 0 a 2 anos a faixa etária prevalente. Os acometimentos mais freqüentes são relativos aos quadros infecciosos, principalmente pulmonares. Os traumas, as cirurgias eletivas ou de urgência e as internações para averiguação diagnóstica ou por agravamento das doenças crônicas aparecem com relativa freqüência, mas contabilizam um número menor de casos. $\mathrm{O}$ período de internação mais freqüente é de 3 a 5 dias, embora haja casos de internação acima de 10 dias e uns poucos que permanecem meses no hospital. Na medida em que o hospital atende à comunidade universitária e à população da região, os usuários da enfermaria pediátrica constituem um grupo heterogêneo no que se refere à rede social de suporte de que desfrutam. Entretanto, destaca-se uma maior freqüência de usuários em situação de significativa vulnerabilidade e com rede de suporte reduzida. Os usuários, em sua maioria, são acompanhados por suas mães durante a internação, embora se observe casos de crianças e adolescentes abrigados, acompanhados ou não por funcionários do abrigo. Existe um grande número de mães jovens que se beneficiariam de um acompanhamento e orientação com relação à ampliação de seus repertórios de interação e cuidado com a criança. Com freqüência encontramos usuários que demandam cuidados especiais seja porque são dependentes de oxigenoterapia, apresentam deficiência física e/ou mental, têm re-internações freqüentes e/ou têm seus quadros deflagrados ou comprometidos por suas precárias condições nutricionais, de moradia e saneamento básico.

Para contemplar as diferentes necessidades dos usuários dos serviços da enfermaria, realizou-se um desenho para o Projeto ACCALANTO a partir de diferentes 'eixos e estratégias de cuidado'. Nesses buscou-se lidar com algumas 
situações-chave que precisavam ser abordadas de formas distintas, criando frentes de trabalho particulares. A primeira era referente ao elevado número de crianças de zero a dois anos internadas por problemas respiratórios na enfermaria. Tal condição se reporta ao alto índice de morbi-mortalidade infantil nessa faixa etária e tem sido alvo de intervenções específicas da atenção básica à saúde. Mesmo assim, há uma demanda ininterrupta de crianças internadas nessa faixa etária para cujos cuidadores, o hospital continua sendo a porta de entrada. A precariedade das condições de vida de algumas dessas crianças com internações repetidas indicam a falta de acesso de suas famílias às políticas sociais e de saúde. Apontam também para uma insuficiência no cuidado que combina falta de informação sobre educação, saúde e desenvolvimento infantil e limitação de repertórios de cuidado.

Foi pensando nessa população que foi desenhado o Projeto Ninar. Visando uma dimensão de escuta e acolhimento a essas condições individuais e sociais, esse projeto busca desenvolver ações de promoção ao cuidado do bebê numa dimensão ampliada. Por um lado, aborda a ampliação dos repertórios de cuidado de mães/pais/ cuidadores, objetivando atender às necessidades do bebê, o fortalecimento do vínculo e de relações afetivas pais/ bebê e o favorecimento de seu desenvolvimento pleno. Por outro, busca trabalhar mães/pais e cuidadores no sentido da apropriação da gestão da vida e do acesso à rede de suporte. Tendo o cotidiano infantil como eixo, o projeto faz uso de oficinas que envolvem conversa, a troca de informações, o uso de cantigas de ninar e de brincadeiras, a confecção de objetos e brinquedos, dentre outros. Acreditase que o Projeto Ninar é propício ao desenvolvimento de ações interdisciplinares e que a realização de projetos compartilhados deva ser fomentada.

A segunda frente de trabalho identificada, e posteriormente denominada de Projeto Aquarela, é referente ao cuidado de crianças e adolescentes cujas condições de saúde são marcadas pela intensidade, cronicidade ou gravidade dos acometimentos que sofrem. Demandam, portanto, intervenções particularizadas com grau elevado de planejamento e controle, dadas a especificidade e a complexidade de sua condição. São encaminhados para o Projeto Aquarela crianças e adolescentes (que):

- apresentem transtornos significativos no desenvolvimento neuropsicomotor ou em situações clínicas que provoquem o não favorecimento deste, e que necessitem de adaptações e cuidados especializados;

- enfrentem dificuldades ou impedimentos na realização de atividades de auto cuidado e de seus interesses ou necessidades cotidianas, demonstrando dificuldade, ausência ou perda de autonomia.

- apresentem quadro de sofrimento psíquico demonstrando apatia, medo excessivo, por situações de dor, labilidade emocional, dificuldade no relacionamento com a família e/ou equipe de cuidado;

- requeiram cuidados especiais no seu pré e pós cirúrgico ou que necessite orientação e acompanhamento pósalta;

- estejam com restrição de contato e não possam circular pela enfermaria, tendo que ficar restritas ao leito ou ao quarto, configurando-se uma situação de isolamento;

- necessitem cuidados especiais, dada a cronicidade e gravidade de suas condições de saúde, podendo envolver a presença de quadro de deficiência grave, problemas cardio-respiratórios, síndromes genéticas ou doenças progressivas;

- com familiares e cuidadores que apresentem dificuldades na relação com a criança e/ ou adolescente na execução de cuidados, no acompanhamento, na compreensão da situação clínica dos mesmos, na organização do cotidiano frente ao cuidado a ser desenvolvido em seus ambientes domésticos.

Como conseqüência, uma característica dessa frente de trabalho é a demanda por uma construção mais estruturada da rede de cuidados e do acesso às redes sociais de suporte. Entretanto, há que se ter em consideração que, devido ao perfil desse hospital em particular, essas internações em geral são transitórias, mesmo quando venham a durar vários meses. Os usuários que continuam a demandar cuidados hospitalares são encaminhados para internação domiciliar ou para hospitais de retaguarda.

O Projeto Aquarela, portanto, tem como foco acolher as necessidades das crianças/adolescentes, de seus pais e cuidadores, resgatando sua cotidianidade e atividade geral, visando à redução dos efeitos adversos do adoecimento $\mathrm{e}$ da hospitalização. Através de metodologias apropriadas e fazendo uso de um repertório variado de atividades que envolvam participação ativa, assistida ou passiva da criança, busca-se favorecer a recuperação da saúde e a manutenção de uma vida de qualidade. Propõe-se também a promoção da ambiência em consonância com a Política Nacional de Humanização.

A terceira frente de intervenção identificada é referente às atividades que podem ser oferecidas ao conjunto de crianças e adolescentes na brinquedoteca e nas áreas de convivência da enfermaria. Este é o foco do Projeto Amarelinha cujo eixo de cuidado visa ofertar, por meio da 
brincadeira, de atividades plásticas e de contação de histórias, uma ampliação ou resgate de aspectos importantes à saúde integral da criança de modo a propiciar uma convivência e cotidianidade diferenciada no espaço hospitalar. Assim, suas atividades são oferecidas para aqueles que podem transitar sem restrição pela enfermaria, como para aqueles, sem restrição de contato, mas que necessitam ser deslocados do seu leito por macas e cadeiras de roda para que possam participar.

Já o Projeto AdoleSer desenvolve, especialmente, ações voltadas à população adolescente. Através do uso de atividades artísticas, corporais e/ou culturais, atende grupos de adolescentes de caráter heterogêneo em relação às origens e características da demanda de seu cuidado. Assume o hospital universitário como um dos contextos de sua intervenção, assim como, desenvolve atividades no Centro de Docência e Pesquisa. Atende em grupos de atividades ou em acompanhamentos individuais, adolescentes em geral e jovens mães ofertando um espaço de re-invenção de si e de projetos de vida por meio de atividades cênicas (teatro dança performance, expressão corporal). Objetiva, ainda, ampliar a rede de sustentação do projeto de maneira a viabilizar uma troca maior entre serviços e atores de projetos voltados para a atenção ao adolescente.

\section{CONSIDERAÇÕES FINAIS}

O Projeto ACCALANTO tem sido construído na interconexão do ensino, da pesquisa e da extensão. Isso faz com que haja um esforço contínuo de busca de interlocução entre o que é essencial nos diferentes domínios que devem agir em consonância e se retroalimentar. O campo da produção do cuidado à saúde tem o desafio de desconstruir o exercício profissional por procedimento para fundar sua ação a partir de uma escuta dos sujeitos em demanda de cuidados integrais. Isso requer programas de ação, ensino e pesquisa diferenciados. Considerando o fato de que o terapeuta ocupacional não protagoniza o papel profissional do curador, a legitimação de sua importância no contexto hospitalar requer a busca de argumentos consistentes que podem ser providos pela pesquisa e pelo relato de experiências. Ao apresentar a construção teórico metodológica do projeto, esse texto buscou contribuir com alguns elementos para o debate. Textos futuros sobre os eixos de cuidado propostos poderão descrevê-los em maior detalhe e apresentar a delicadeza e a complexidade que permeiam as ações de um terapeuta ocupacional junto a crianças e adolescentes sob cuidados clínicos e cirúrgicos.

GALHEIGO, S. M.; ANGELI, A. A. C. de. Occupational therapy and the integral health care of children and adolescents: the construction of the Project ACCALANTO. Rev. Ter. Ocup. Univ. São Paulo, v. 19, n. 3, p. 137-143, set./dez. 2008.

ABSTRACT: This paper presents the Project ACCALANTO, which develops activities of teaching, research and assistance in the realm of occupational therapy and the child and adolescent care, via the perspective of integral and humanized care and the integral protection of childhood and youth. This project proposes to contribute to the development of knowledge and practices in occupational therapy, supported by the principles of an amplified clinic which recognizes e holds the singular needs of children and adolescents and their families/caregivers, caused by the process of getting ill and hospitalized and by the conditions of social vulnerability to which they are exposed. The paper presents the rationale and the theoretical/methodological framework and, the main care axis and strategies used in a child ward, namely: (i) the Ninar - comprehension and holding towards the needs of children from birth to 2 years old and their parents', seeking to develop initiatives of care promotion in an amplified dimension; (ii) the Aquarela - care for children and adolescents whose health conditions are marked by the intensity, gravity or chronicity, requiring especial intervention with high level of planning and control; (iii) the Amarelinha provision of a significant co-existence and daily life in the hospital setting through the use of activities for the users altogether either in the toy library or in the common rooms of the ward; (iv) the AdoleSer - care provided for adolescents and young mothers in order to offer a place for reinvention of themselves and their life projects.

KEY-WORDS: Comphrehensive health care. Occupational therapy. Occupational therapy department, hospital. Child care. Health-disease process. Activities of daily living. 


\section{REFERÊNCIAS}

BRASIL. Ministério da Saúde. Secretaria Executiva, Núcleo Técnico da Política Nacional de Humanização. HumanizaSUS: Política Nacional de Humanização. Brasília: Ministério da Saúde, 2004a.

BRASIL. Ministério da Saúde. Secretaria de Atenção à Saúde. Departamento de Ações Programáticas Estratégicas. Agenda de compromissos para a saúde integral da criança e redução da mortalidade infantil. Ministério da Saúde, Secretaria de Atenção à Saúde, Departamento de Ações Programáticas Estratégicas. Brasília: Ministério da Saúde, 2004b.

BRASIL. Ministério da Saúde. Secretaria de Atenção à Saúde. Saúde integral de adolescentes e jovens: orientações para a organização de serviços de saúde. Brasília: Editora do Ministério da Saúde, 2005. (Série A. Normas e Manuais Técnicos).

CASTRO, E. D. de. Inscrições da relação terapeuta-paciente. Rev. Ter. Ocup. Univ. São Paulo, v. 16, n. 1, p. 14-21, 2005.

CECÍLIO, L.; MERHY, E. E. Integralidade do cuidado como eixo da gestão hospitalar. In: PINHEIRO, R.; MATTOS, R.; CAMARGO Jr., K. R. (org.). Construção da integralidade, cotidiano, saberes e práticas em saúde. Rio de Janeiro: UERJIMS Abrasco, 2003..
FEUERWERKER, L.; CECÍLIO, L. O hospital e a formação em saúde: desafios atuais. Cien Saude Coletiva., v. 12, n. 4, p. 965-971, 2007.

GALHEIGO, S. M. Terapia ocupacional, a produção do cuidado em saúde e o lugar do hospital: reflexões sobre a constituição de um campo de saber e prática. Rev. Ter. Ocup. Univ. São Paulo, v. 18 , n. 1, p. 20-28, 2008.

GAlHeigo, S. M.; ANGELI, A. C. Projeto ACCALANTO: a interface com a extensão. São Paulo: Universidade de São Paulo, 2007. mimeo

MERHY, E. Em busca do tempo perdido: a micropolítica do trabalho vivo em saúde. In: MERHY, E.; ONOCKO, R. Agir em saúde: um desafio para o público. São Paulo: Hucitec, 1997a. p. 71-112.

MERHY, E. et al. Em busca de ferramentas analisadoras das tecnologias em saúde: a informação e o dia a dia de um serviço interrogando e gerindo trabalho em saúde. In: MERHY, E.; ONOCKO, R. Agir em saúde: um desafio para o público. São Paulo: Hucitec, 1997b. p. 71-112.

SANT'ANNA, D. Corpos de passagem - ensaios sobre a subjetividade contemporânea. São Paulo: Estação Liberdade, 2001. 